\title{
Evaluation of solid (disc diffusion)- and liquid (turbidity)-phase antibiogram methods for clinical isolates of diarrheagenic $E$. coli and correlation with efflux
}

\author{
Alagiachidambaram Alagumaruthanayagam ${ }^{1}$, Asalapuram R Pavankumar ${ }^{1}$, Thangammal K Vasanthamallika ${ }^{2,3}$ \\ and Krishnan Sankaran ${ }^{1}$
}

\begin{abstract}
Multiple drug resistance (MDR) in bacteria causes higher mortality and morbidity, complicates treatment and increases healthcare outlay. With no new-generation antibiotics in sight, its rapid spread through the environment poses grave danger. Therefore, rapid detection to identify effective antibiotics and to prevent their indiscriminate use is imperative. However, the widely used clinical method for antibiogram, the Kirby-Bauer disc-diffusion method (DDM), requires 2-3 days, has inherent shortcomings of solid-phase assays and is not suitable for high-throughput operations. In our research on MDR associated with childhood diarrhea, we determined the antibiogram of 73 clinical diarrheagenic Escherichia coli strains using both the DDM and the more reliable liquid turbidity method (LTM) performed in 96-microwell plates. The results were further correlated with a dye-exclusion efflux assay using fluorescein diacetate. Although LTM is apparently superior in saving critical time, suitability to highthroughput operations and reliability, we found that the serious shortcomings of DDM could be effectively countered by just doubling the dosage of antibiotics currently used in discs or by using two discs in place of one. With 48 of the 49 MDR strains being positive for efflux and the 12 strains 'susceptible' to all the antibiotics being negative, the efflux assay could be useful as an integral component of the antibiogram test or for additional confirmation. The presence of $65 \%$ of MDR strains among diarrheagenic $E$. coli is a matter of serious concern, although most of them could be treated with either Gentamycin or
\end{abstract} Amikacin, as is the practice by experience.

The Journal of Antibiotics (2009) 62, 377-384; doi:10.1038/ja.2009.45

Keywords: antibiogram; antibiotics; efflux; fluorescence; Kirby-Bauer disc-diffusion method; liquid turbidity method; multidrugresistant E. coli.

\section{INTRODUCTION}

Multiple drug resistance (MDR) in bacteria is increasingly and widely reported. Even common pathogens such as Salmonella spp. are developing resistance to multiple antibiotics and are complicating treatment procedures. ${ }^{1}$ Globally, the morbidity and mortality rates due to MDR in bacteria affect billions of people, and the expenditure incurred exceeds $\$ 19$ billion in the United States and the United Kingdom annually. ${ }^{2}$ It contributes to $10-31 \%$ of mortality in hospitals. ${ }^{3}$

Antibiotic resistance is caused by enzymatic inactivation (for example, $\beta$-lactamases), or by the mutation of target (for example, gyrases) or by active efflux, ${ }^{4-6}$ which is an important diagnostic target providing nonspecific resistance to multiple antibiotics. ${ }^{7}$ Genetic transfer of resistance among microbes in the environment ${ }^{8,9}$ calls for the prudent use of antibiotics ${ }^{10}$ with reliable antibiograms. In spite of limitations, such as formation of concentration gradients in solid-phase assays and the delay of 2-3 days for obtaining results, the Kirby-Bauer disc-diffusion method (DDM) is the only widely used clinical method. Other techniques such as colorimetric, fluorimetric or flow cytometric methods and PCR are unsuitable because of cost, labor or skill. ${ }^{11}$ Such limitations force clinicians to select improper antibiotics for early diagnosis and treatment. In this regard, the microculture method that is performed routinely in 96well plates for screening microbes and their metabolites is more appealing.

In our study on childhood diarrheagenic pathogens, it is observed that MDR strains are normally associated with severe cases, and we are concerned about the loss of critical time in selecting effective antibiotics.

${ }^{1}$ Centre for Biotechnology, Anna University, Chennai, Tamilnadu, India and ${ }^{2}$ Department of Pediatrics, Institute of Child Health and Hospital for Children, Chennai, Tamilnadu, India

${ }^{3}$ Current address: Division of Pediatrics, Madras Medical Mission, Chennai, Tamilnadu, India

Correspondence: Professor K Sankaran, Centre for Biotechnology, Anna University, Sardar Patel Road, Chennai 600025, Tamilnadu, India.

E mails: ksankran@yahoo.com or ksankaran@annauniv.edu

Received 2 April 2009; revised 19 May 2009; accepted 21 May 2009 
Table 1 Antibiotics used and their respective doses

\begin{tabular}{lcc}
\hline Antibiotics & $\begin{array}{c}\text { Kirby-Bauer disc-diffusion } \\
\text { method (dose in } \mu g \text { ) }\end{array}$ & $\begin{array}{c}\text { Liquid turbidity method } \\
\text { (dose in } \mu g \text { ) }\end{array}$ \\
\hline Amikacin & $30 / 60^{\mathrm{a}}$ & 2 \\
Gentamycin & $10 / 20^{\mathrm{a}}$ & 2 \\
Kanamycin & $30 / 60^{\mathrm{a}}$ & 6 \\
Nalidixic acid & 30 & 3 \\
Ampicillin & 10 & 20 \\
Ceftazidime & $30 / 60^{\mathrm{a}}$ & $2 / 4^{\mathrm{a}}$ \\
Cephotaxime & $30 / 60^{\mathrm{a}}$ & $4 / 8^{\mathrm{a}}$ \\
\hline
\end{tabular}

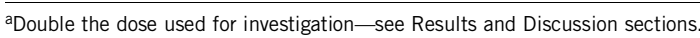

In particular Escherichia coli, an emerging pathogen with a variety of virulent attributes, contributes to $20-30 \%$ of childhood mortality globally. ${ }^{12,13}$ In this study, we determined the antibiograms (against commonly prescribed antibiotics for diarrhea) of 73 clinical isolates of E. coli using both DDM and the microplate-based liquid turbidity method (LTM). This comparative study is also meant to address the limitations of DDM over LTM and find possible simple solutions to enhance its reliability. Correlation of the MDR phenotype with the efflux of fluorescein diacetate (FDA) in line with their known association was carried out to probe the utility of efflux assays in the early detection of MDR. This study has provided a reliable estimate of MDR among childhood diarrheagenic E. coli, which is an important emerging pathogen.

\section{MATERIALS AND METHODS}

\section{Instruments and standard procedure used}

OD of bacterial cultures was measured using Multiscan (Thermo Bioanalysis Company, Labsystems Oy, Vantee, Finland). Intensity of fluorescence was measured in the form of relative fluorescence units (RFUs) using Fluroscan (Thermo Bioanalysis Company). Drug susceptibility was determined using the Kirby-Bauer DDM by measuring the zone of clearance.

The selected antibiotics comprised three groups, namley aminoglycosides (Amikacin, Kanamycin and Gentamycin); penicillins (Ceftazidime, Cephotaxime and Ampicillin) and quinolones (Nalidixic acid). The antibiotic-impregnated discs with a uniform disc diameter of $8 \mathrm{~mm}$ for DDM were procured from Himedia Laboratories (Mumbai, Maharashtra, India). Antibiotics used in LTM were procured from the following different indigenous suppliers: Amikacin (Aristo Pharma, Mumbai, India), Gentamycin (Fulford Pharma, Mumbai, India), Kanamycin (Macleods Pharma, Mumbai, India), Ampicillin (Ranbaxy Pharma, Gurgaon, India), Cephotaxime (Lupin Labs, Mumbai, India), Ceftazidime (GlaxoSmithKline, Mumbai, India) and Nalidixic acid (Ranbaxy Pharma). The doses of different antibiotics in LTM were calculated according to the following formula ${ }^{14}$

$$
W=(1000 / P) * V * C
$$

where $W$ is the weight of antibiotic to be dissolved in $\mathrm{V}$, the volume required; $P$, potency of the antibiotic base; and $C$, final concentration of solution. Table 1 represents the final doses of antibiotics used in both the methods. Half-lives of all antibiotics except Nalidixic acid $(6 \mathrm{~h})$ and Cephotaxime $(0.9 \mathrm{~h})$ are in the range of $2-3$ h. FDA (Molecular Probes, Invitrogen, Eugene, OR, USA) was used to detect efflux activity.

Collection and identification of clinical diarrheagenic $E$. coli isolates Escherichia coli strains were isolated from stool samples of children, who were hospitalized because of acute or persistent diarrhea at the Institute of Child Health and Hospital for Children (ICH and HC, Chennai, Tamilnadu, India). E. coli strains were identified by conducting standard biochemical tests such as IMViC (Indole, Methyl Red, Voges-Proskauer and Citrate) ${ }^{15}$ and were confirmed by PCR. ${ }^{16}$
Preparation of Luria Bertani broth and Mueller-Hinton agar plates Luria Bertani (LB) broth and Mueller-Hinton agar (MHA, Himedia) plates were used to perform antibiograms of LTM and DDM, respectively. LB was prepared by dissolving $10 \mathrm{~g}$ of Tryptone (Himedia), $5 \mathrm{~g}$ of yeast extract (Himedia) and $10 \mathrm{~g}$ of sodium chloride (Merck, Mumbai, India) in one liter of distilled water, and the $\mathrm{pH}$ was adjusted to 7.2 with $1 \mathrm{~m}$ sodium hydroxide (Merck). MHA plates were prepared by dissolving $38 \mathrm{~g}$ of MHA in one liter of distilled water, sterilized and cooled to $\sim 45^{\circ} \mathrm{C}$, and $20 \mathrm{ml}$ of the molten agar was poured into pre-sterilized petri plates. The plates were checked for sterility by incubating them at $37^{\circ} \mathrm{C}$ for $6-7 \mathrm{~h}$ before experimentation. ${ }^{14,17}$

\section{Kirby-Bauer DDM}

Approximately $10^{8}$ cells ( 1 OD corresponding to $10^{9}$ cells $/ \mathrm{m}^{18}$ ) of E. coli were spread-plated on the above-prepared MHA plates. Antibiotic discs were placed in duplicates and incubated at $37^{\circ} \mathrm{C}$ for $16-18 \mathrm{~h}$ for zone formation. Zones of inhibition measured as mean diameter were categorized as 'susceptible' (S), 'intermediary' (I) and 'resistant' (R) according to Clinical and Laboratory Standards Institute (CLSI) standards. ${ }^{19}$ DDM with double the dose of antibiotic concentration was performed by stacking two discs (instead of one) on MHA plates. We resorted to increase the doses of the antibiotics retaining the same CLSI standards for the zone of clearance.

\section{Liquid turbidity method}

Approximately $10^{5}$ cells were cultured by shaking at 170 r.p.m. at $37^{\circ} \mathrm{C}$ in $200 \mu \mathrm{l}$ of LB with calculated amounts of antibiotics (in duplicates) contained in each well of a 96-well microtiter plate (Laxbro Bio-Medical Aids, Pune, India) (Table 1). The turbidity measured at hourly time intervals for $8 \mathrm{~h}$ was plotted and compared with control (without antibiotic). On the basis of OD at $595 \mathrm{~nm}$ (measured using Multiscan, Thermo Bioanalysis Company), the strains were categorized with respect to their susceptibility as $\leqslant 0.05$, 'susceptible;' $0.05-$ 0.15 , 'intermediate;' $\geqslant 0.15$, 'resistant.' Figure 1 shows the distinct growth pattern for the 'resistant' and 'intermediate' types for a set of representative strains. The clinical isolates found 'resistant' to three or more antibiotics were scored as MDR phenotypes. ${ }^{14}$

\section{Detection of efflux using FDA}

Fluorescein diacetate is a known marker used to study bacterial viability. ${ }^{20,21}$ The dye was added to the medium at a concentration of $50 \mu \mathrm{M}$ just $30 \mathrm{~min}$ before harvesting the cells, to avoid interference due to a spontaneous hydrolysis of the dye by the medium. ${ }^{22}$ Viable 'resistant' bacteria are able to convert the passively transported dye from non-fluorescent form (FDA) to its charged acid form, fluorescein, and efflux it actively using a pump mechanism. Therefore, fluorescence in the medium indicates the operation of efflux mechanism and hence viability. Fluorescence in the spent medium obtained after centrifugation $(6000 \mathrm{~g}, 1 \mathrm{~min})$ was measured using a fluorimeter (Fluroscan, Thermo Bioanalysis Company) in a microtiter plate ${ }^{23}$ and then correlated with the antibiogram results of LTM.

\section{RESULTS}

For a clinically relevant investigation, the efficacy of the popular clinical antibiogram method (DDM) was compared with that of the turbidity measurement method (normally used in research) among drug-resistant diarrheagenic E. coli, which is an emerging threat in pediatric care. This knowledge could be useful in the management of pediatric diarrhea due to MDR strains. Among the 73 strains used in this study, 69 were isolated from children with persistent diarrhea, 3 strains (E. coli A5, E. coli B5 and E. coli C2) were reference strains and 1 was the laboratory strain E. coli $(\mathrm{DH} 5 \alpha)$.

Lack of correlation in antibiogram results between DDM and LTM The degree of antibiotic susceptibility of these isolates to the first-line antibiotics was determined using both DDM and LTM and scored as 'susceptible,' 'intermediate' and 'resistant' (Table 2). A fair correlation between the two methods can be observed in all ' $\mathrm{S}$ ' and ' $\mathrm{R}$ ' categories 


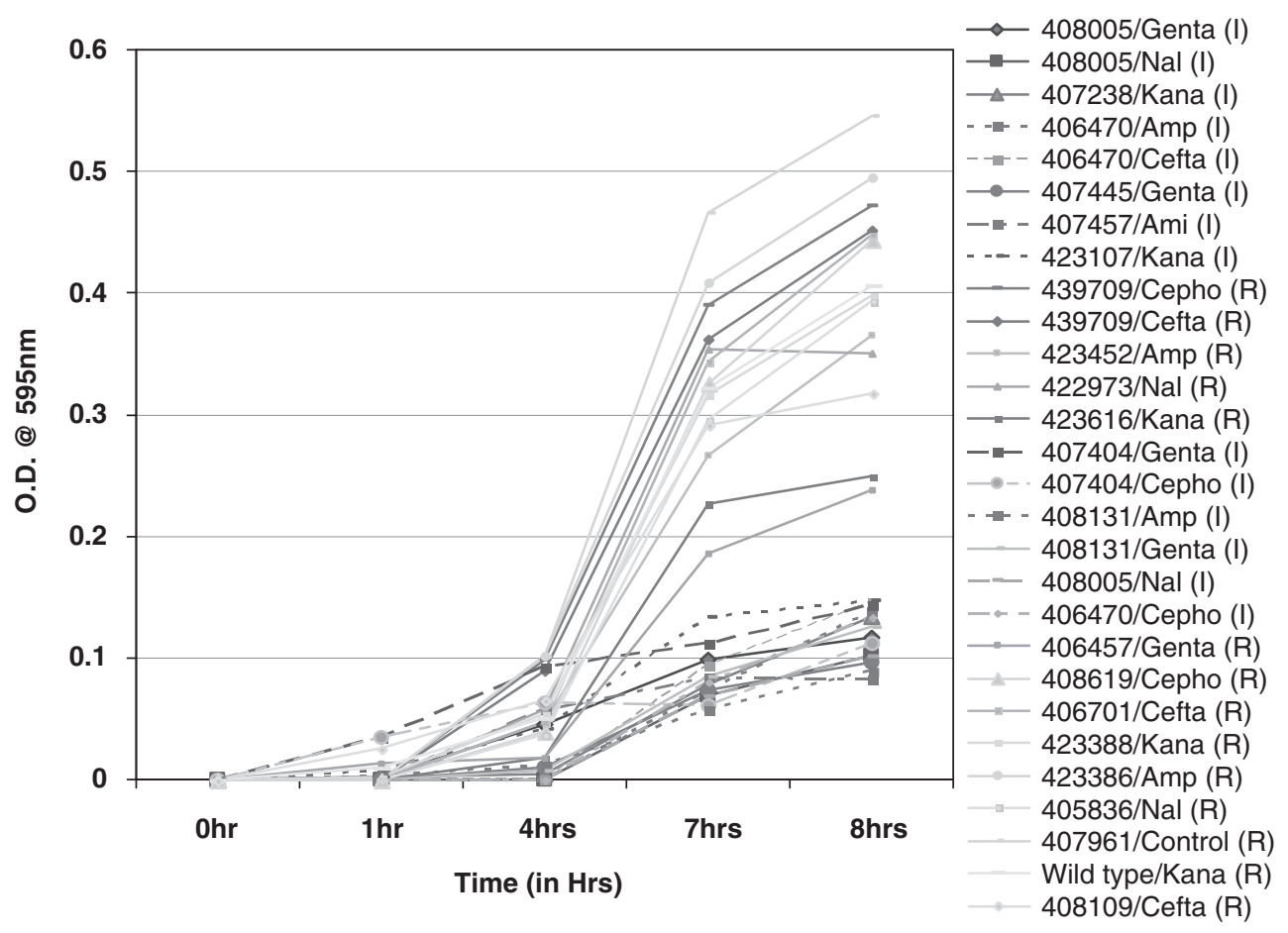

Figure 1 The graph shows a distinct difference in growth between 'Intermediates' (O.D. 0.05-0.15) and 'Resistant' ( $\geqslant 0.15)$ strains.

of Nalidixic acid, Amikacin and Ceftazidime; in particular, the lastmentioned drug showed the best correlation; The 'intermediary' in these cases were correlating poorly, probably because of the subjectivity in manual measurement in DDM. Generally, DDM tended to score less ' $\mathrm{S}$ ' types compared with LTM. In contrast, the ' $\mathrm{R}$ ' types were present more in DDM and less in LTM. DDM scored more 'intermediary' for Amikacin, Kanamycin and Cephotaxime, and less for others, in comparison with LTM.

The number of strains identified by DDM as 'susceptible' to Ampicillin, Ceftazidime and Cephotaxime, all belonging to the same penicillin group was quite variable, and in the 'intermediary' category, the number was particularly high for Cephotaxime. LTM, on the other hand, showed similar numbers for the whole group in all the categories. A better correlation was observed between both the methods only in the ' $\mathrm{R}$ ' category. In the group of aminoglycosidic antibiotics, only six strains, in the case of Kanamycin, and nearly half of the strains in the case of Amikacin and Gentamycin were found to be 'susceptible' by DDM, whereas LTM showed uniformly high scores (80-90\%) in this category. DDM scored about a third of the strains to be 'resistant,' whereas, in contrast, either none or only a few were scored by LTM. To rule out the possibility that the quality of commercial antibiotic disc could affect the results, we even used homemade discs that contained the specified amount of antibiotics from the same stock used for LTM. We did not find any significant difference in the results (data not shown).

\section{Physicochemical and stability factors of antibiotics affect DDM results}

To account for the above-mentioned contrasting behavior and to identify a better method, especially in view of the limitations of solidphase assays compared with liquid-phase assays, we have compiled in Table 3 various properties of the antibiotics ${ }^{24,25}$ that could plausibly affect the zone of clearance in DDM. The contrasting ' $\mathrm{S}$ ' scores in the case of Kanamycin by DDM (6/73) and LTM (63/73) could be
Table 2 Antibiotic susceptibility: comparison of disc-diffusion method (DDM) and liquid turbidity method (LTM)

\begin{tabular}{|c|c|c|c|c|c|c|}
\hline \multirow{2}{*}{$\begin{array}{l}\text { Susceptibility } \\
\text { Antibiotics }\end{array}$} & \multicolumn{2}{|c|}{$S$ (Nos.) } & \multicolumn{2}{|c|}{ I (Nos.) } & \multicolumn{2}{|c|}{$R$ (Nos.) } \\
\hline & $D D M$ & LTM & $D D M$ & LTM & $D D M$ & LTM \\
\hline Amikacin & 35 & 72 & 18 & 01 & 20 & 00 \\
\hline Gentamycin & 39 & 60 & 08 & 11 & 26 & 02 \\
\hline Kanamycin & 06 & 63 & 35 & 03 & 32 & 07 \\
\hline Nalidixic acid & 10 & 14 & 04 & 10 & 59 & 49 \\
\hline Ampicillin & 14 & 22 & 04 & 10 & 55 & 41 \\
\hline Ceftazidime & 30 & 25 & 03 & 12 & 40 & 36 \\
\hline Cephotaxime & 08 & 32 & 25 & 10 & 40 & 31 \\
\hline
\end{tabular}

Abbreviations: I, intermediate; R, resistance; S, Susceptible.

Nos. represents the number of strains.

attributed to the higher minimal inhibitory concentration ratios (MIC) $\left(8 \mu \mathrm{g} \mathrm{ml}^{-1}\right)$ of the antibiotic requiring higher concentrations in the killing zone. Hence, most of the strains would have been distributed either as ' $I$ ' or ' $R$ ' in DDM. In another instance, it is well known that DH5 $\alpha$ is 'susceptible' to all antibiotics other than Nalidixic acid, but the zone of clearance for Ceftazidime under our experimental condition was found to be $14 \mathrm{~mm}$, indicating that it was 'resistant.' This anomaly may be attributable to its higher molecular weight (637) and sparingly soluble nature, both limiting drug diffusion. Instead of adjusting for all these complex factors that affect the antibiotic availability to form proper zones of clearance, we resorted to increasing the doses of the antibiotics retaining the same CLSI standards for the zone of clearance.

As can be seen in Table 4, doubling the dose by stacking two discs increased the number of strains 'susceptible' to aminoglycosides. All the 73 strains were now found to be 'susceptible' to Amikacin compared with 35 originally; 69 were 'susceptible' for Gentamycin compared with 39 before. For Kanamycin, the numbers increased 
Table 3 Properties of different antibiotics that could influence antibiogram

\begin{tabular}{|c|c|c|c|c|c|c|c|}
\hline $\begin{array}{l}\text { Properties } \\
\text { Antibiotics }\end{array}$ & $\begin{array}{l}\text { Molecular } \\
\text { formula }\end{array}$ & $\begin{array}{l}\text { Molecular } \\
\text { weight }\end{array}$ & $\begin{array}{l}\text { Solubility } \\
\text { in water }\end{array}$ & $\begin{array}{c}M I C \\
\left(\text { in } \mu g \mathrm{ml}^{-1}\right)\end{array}$ & $\begin{array}{l}\text { Observed zone of clearance } \\
\quad \text { for } \mathrm{DH} 5 \alpha(\text { in } \mathrm{mm})\end{array}$ & $\begin{array}{l}\text { Half-life } \\
\text { (in hours) }\end{array}$ & $\begin{array}{l}\text { Factors influencing the } \\
\text { zone of clearance }\end{array}$ \\
\hline Amikacin & $\mathrm{C}_{22} \mathrm{H}_{43} \mathrm{~N}_{5} \mathrm{O}_{13}$ & 781.75 & Freely soluble & 10 & 19 & $2-3$ & Higher molecular weight, high solubility, high MIC \\
\hline Kanamycin & $\mathrm{C}_{18} \mathrm{H}_{36} \mathrm{~N}_{4} \mathrm{O}_{11}$ & 484 & Freely soluble & 8 & 23 & $2-4$ & High solubility, moderate MIC \\
\hline Nalidixic acid & $\mathrm{C}_{12} \mathrm{H}_{12} \mathrm{~N}_{2} \mathrm{O}_{3}$ & 232.24 & $\begin{array}{l}\text { Sparingly } \\
\text { soluble }\end{array}$ & 5 & 11 & $6-7$ & $\begin{array}{l}\text { Low molecular weight, low solubility, moderate MIC, } \\
\text { higher half life }\end{array}$ \\
\hline Cephotaxime & $\mathrm{C}_{16} \mathrm{H}_{16} \mathrm{~N}_{6} \mathrm{O}_{7} \mathrm{~S}_{2}$ & 477.44 & Freely soluble & 4 & 19 & $0.9-1.7$ & High solubility, low-moderate MIC, lower half-life \\
\hline
\end{tabular}

The MIC values are against Gram-negative bacteria, in general E. coli spp. and the reported half-life is the time of plasma elimination in humans. The values are adopted from British Pharmacopoeia ${ }^{24}$ and United States Pharmacopoeia. ${ }^{25}$

from a mere 6 for a single dose to 50 with a corresponding shift from 'I' and 'R' phenotypes. In the case of Cephotaxime of the cephalosporin group, doubling the dose brought many from the ' $\mathrm{I}$ ' and ' $\mathrm{R}$ ' categories into ' $\mathrm{S}$ ' (from 8 to 49). This is perhaps justified by the labile nature of the drug with a lower half-life (Table 3 ) and therefore, the increased dose could have compensated the loss of potency. For the same reason, doubling the concentration of Cephotaxime in LTM, drastically reduced the ' $\mathrm{R}$ ' types (from 31 to 13 ) with a corresponding increase in ' $\mathrm{I}$ ' (3) and ' $\mathrm{S}$ ' (15) types. In the case of Ceftazidime, a related member in the group with better half-life, doubling the dose did not alter the numbers significantly. Among 36 'resistant' phenotypes only 4 turned out to be 'susceptible' and 2 were 'intermediate,' the rest remained as 'resistant' forms.

\section{MDR phenotype detection by antibiogram methods and efflux matched well}

A novel adaptation of bacteria to counter antibiotic pressure is in using an efflux pump to effectively throw out the antibiotic. In Table 5, the antibiogram of individual strains by both the methods (DDM and LTM modified for the dosage) and their efflux activity has been shown. The efflux activity, as indicated by the zero or low background RFU value, was clearly absent in 'susceptible' cases. However, the absolute RFU, did not clearly indicate the level of resistance, either 'I' or 'R.' There were cases of 'resistant' phenotypes with moderate RFU and 'intermediary' with high RFU. Without correlating the RFU quantities with ' $\mathrm{I}$ ' or ' $\mathrm{R}$ ' phenotypes, anything above the background level of fluorescence was considered efflux positive. Table 6 shows that several strains were 'resistant' to $\geq 3$ antibiotics, the common two being Nalidixic acid and Ampicillin, normally avoided in the treatment of diarrhea.

There was a good correlation between the efflux and MDR phenotypes seen in LTM, as summarized in Table 6; 'intermediary' was also considered as 'resistant' for compilation. To detect the efflux in strains that were 'susceptible' to all antibiotics, we had to grow them in the absence of antibiotics and the strains were found to be efflux negative. The results of efflux and antibiogram reflect the documented association of efflux with MDR phenotypes. The fluorescence-based efflux detection could be a good addition to the tools for antibiogram. In total, 13 strains were found to be 'resistant' to only one or two antibiotics, mostly to Nalidixic acid and Ampicillin, but they were efflux positive. This perhaps indicates that the dye efflux might be occurring through a pump system not involved in antibiotic efflux. ${ }^{21-23}$ The only case of MDR positive but efflux negative, as seen in strain 407457 (Table 5), could possibly contain an enzymatic degradation mechanism for $\beta$-lactamases with a broader specificity. ${ }^{4,5}$
Table 4 Susceptible, intermediary and resistant types based on double the dose of antibiotics in DDM compared with the results of DDM single dose and LTM

\begin{tabular}{llll}
\hline & \multicolumn{3}{c}{ Double disc/single disc (LTM) } \\
\cline { 2 - 4 } Antibiotics & Susceptible (S) & Intermediate (I) & Resistant (R) \\
\hline Amikacin & $73 / 35(72)$ & $00 / 18(01)$ & $00 / 20(00)$ \\
Gentamycin & $69 / 39(60)$ & $03 / 08(11)$ & $01 / 26(02)$ \\
Kanamycin & $50 / 06(63)$ & $06 / 35(03)$ & $17 / 32(07)$ \\
Ceftazidime & $31 / 30(29 / 25)$ & $09 / 03(14 / 12)$ & $33 / 40(30 / 36)$ \\
Cephotaxime & $49 / 08(47 / 32)$ & $18 / 25(13 / 10)$ & $06 / 40(13 / 31)$
\end{tabular}

Abbreviations: DDM, disc-diffusion method; LTM, liquid turbidity method.

The values in parentheses show the score by LTM, which, in the case of Cephotaxime and Ceftazidime, had double the antibiotic dose; the first number corresponds to double dose.

\section{DISCUSSION}

In current clinical practice in India, diarrhea, which is refractory to oral rehydration therapy, is treated using first-line antibiotics, such as cotrimoxazole, or cephalosporins, such as cephalexin, cefazolin and Nalidixic acid, in outpatients. Third-generation cephalosporins such as cefoperazone, Ceftazidime or cefixime are used to treat severe forms of diarrhea. More potent drugs such as cancomycin, methicillin, imipenem are used when diarrhea is associated with other systemic infections, according to the severity of disease. ${ }^{13}$ In spite of the prevalence of MDR strains associated with various infectious diseases including diarrhea, there is no effective clinical tool other than the time-consuming DDM to quickly determine the antibiogram of a pathogen in order to initiate the correct antibiotic therapy or apply early course correction. A lack of correlation between antibiogram results of 73 strains obtained by DDM and LTM prompted us to takeup this study to evaluate superior methods, as it affects the efficacy of treatment. Furthermore, the saving of precious treatment time by adapting to quicker LTM compared with DDM, as well as inherent limitations of solid-phase assays compared with those of liquid-phase assays encouraged us to investigate the former for clinical utility. As the MDR phenotype is increasingly contributed by a broad-spectrum efflux mechanism, we have also incorporated an FDA-based viability assay in LTM for detecting efflux.

\section{Double the antibiotic dose improves the performance of DDM} Overall, it was found that DDM was biased toward 'R' type and scored fewer ' $S$ ' types. This is perhaps attributable to various physicochemical properties ${ }^{24,25}$ of these antibiotics such as molecular weight, water solubility, MIC, half-life etc., in determining the zone of clearance. 
Table 5 Compilation of the results of $73 \mathrm{E}$. coli strains with respect to the methods of determining antibiogram (DDM and LTM modified for the dosage)

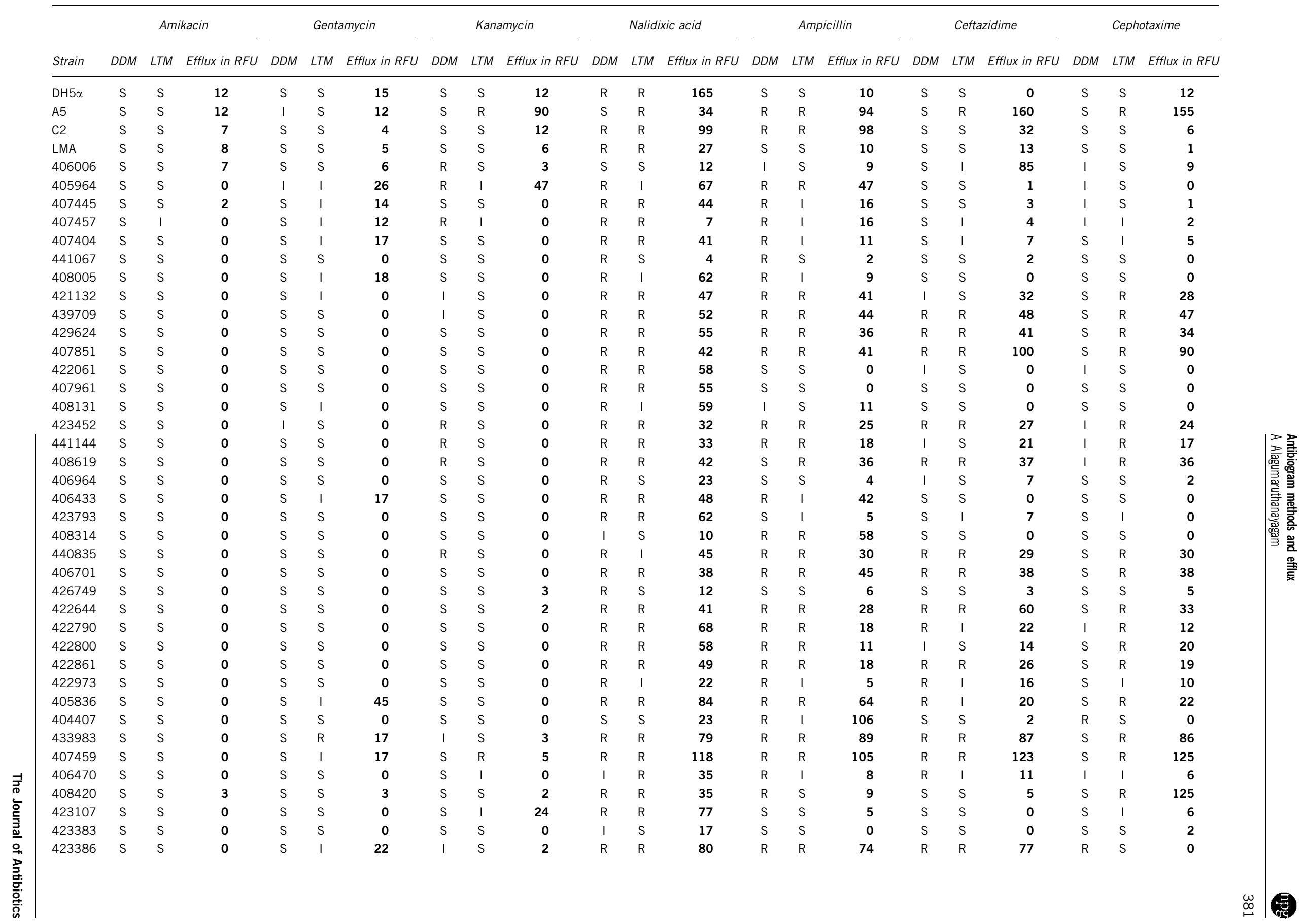


Table 5 Continued

Amikacin

Gentamycin

Kanamycin

Nalidixic acid

Ampicillin

Ceftazidime

Cephotaxime

Strain DDM LTM Efflux in RFU DDM LTM Efflux in RFU DDM LTM Efflux in RFU DDM LTM Efflux in RFU DDM LTM Efflux in RFU DDM LTM Efflux in RFU DDM LTM Efflux in RFU

$\begin{array}{llllllllllll}423427 & S & S & 2 & S & S & 3 & S & S & 17 & R & R\end{array}$

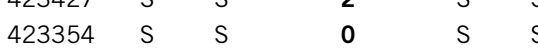

423314 S $S$

423587 S S

423798 S S

423616 S S

402746 S S

408027 S $S$

408109 S S

423892 S $S$

423394 S $S$

408619 S S

406319 S S

423212 S S

423383 S S

423396 S S

423402 S S

407238 S S

406012 S S

407783 S S

408109 S S

407998 S S

423388 S S

433864 S S

$441220 \mathrm{~S} \quad \mathrm{~S}$

406457 S S

406484 S S

EPECl S S

$\begin{array}{lll}\text { EPEC2 } & S & S \\ 403396 & S & S\end{array}$

. Eoli, Escherichia coli; LTM, liquid turbidity method.

Bold values indicate effluxing ability of the bacteria.

\begin{tabular}{|c|c|c|c|c|c|c|c|c|c|c|}
\hline 61 & $\mathrm{R}$ & $R$ & 46 & $\mathrm{R}$ & $\mathrm{R}$ & 132 & $S$ & S & 0 & \\
\hline 62 & $\mathrm{R}$ & $\mathrm{R}$ & 41 & $\mathrm{R}$ & $\mathrm{R}$ & 43 & $\mathrm{R}$ & $\mathrm{R}$ & 78 & \\
\hline 46 & $R$ & $\mathrm{R}$ & 45 & $\mathrm{R}$ & $\mathrm{R}$ & 39 & $S$ & $\mathrm{R}$ & 38 & \\
\hline 46 & $\mathrm{R}$ & $R$ & 39 & $\mathrm{R}$ & $R$ & 36 & $S$ & $R$ & 35 & \\
\hline 40 & $R$ & $\mathrm{R}$ & 34 & $\mathrm{R}$ & $\mathrm{R}$ & 38 & $\mathrm{R}$ & $\mathrm{R}$ & 30 & \\
\hline 62 & $S$ & $S$ & 1 & $\mathrm{~S}$ & $S$ & 0 & $\mathrm{R}$ & $\mathrm{R}$ & 30 & \\
\hline 67 & $S$ & $S$ & 5 & I & 1 & 132 & $S$ & $\mathrm{R}$ & 30 & \\
\hline 12 & 1 & $S$ & 1 & S & $S$ & 5 & S & S & 0 & \\
\hline 62 & $\mathrm{R}$ & $\mathrm{R}$ & 41 & $\mathrm{R}$ & $\mathrm{R}$ & 50 & $S$ & S & 0 & \\
\hline 130 & $\mathrm{R}$ & $S$ & 12 & $\mathrm{R}$ & $\mathrm{R}$ & 343 & $S$ & S & 2 & \\
\hline 15 & $S$ & $S$ & 0 & $\mathrm{~S}$ & $S$ & 2 & 1 & $\mathrm{R}$ & 43 & \\
\hline 108 & $\mathrm{R}$ & $S$ & 12 & $\mathrm{R}$ & $\mathrm{R}$ & 354 & $S$ & $\mathrm{~s}$ & 35 & \\
\hline 7 & 1 & $S$ & 3 & $S$ & S & 7 & $S$ & $\mathrm{~s}$ & 0 & \\
\hline
\end{tabular}


Table 6 Correlation between MDR and efflux

\begin{tabular}{lcccc}
\hline & \multicolumn{2}{c}{ MDR positive } & & MDR-negative/efflux positive \\
\cline { 2 - 3 } Phenotype & Efflux positive & Efflux negative & & All susceptible \\
\hline LTM/efflux & $49 / 48$ & $49 / 1$ & $12 / 12$ & Resistant to any one or two antibiotics \\
\hline
\end{tabular}

LTM, liquid turbidity method.

MDR positive-resistant to three or more antibiotics in LTM; Efflux positive-relative fluorescence unit (RFU) > 15 .

For different antibiotics, the zone of clearance for ' $R$ ' ranges from 12 to $14 \mathrm{~mm}$ across the disc. This leaves only $2-3 \mathrm{~mm}$ annular width in the clearance zone and, at this lower scale, the errors in reading could be very high, especially given the smallest reading in a ruler to be $1 \mathrm{~mm}$. Fairly uniform cutoff of $12-14 \mathrm{~mm}$ for different antibiotics used in this study seems to be artifactual, given the wide variations in solubility (from being freely soluble to sparingly soluble), molecular size (232-760) and MIC (1-22). ${ }^{24,25}$ Although MHA is popular for DDM, parameters such as $\mathrm{pH}$ (that influences the potency of antibiotics such as aminoglycosides, quinolones, tetracyclines and macrolides), moisture content of the media, concentration of thymine or thymidine and variation in divalent cations have been reported to result in variable or unusual zone of clearances (in antibiotics such as aminoglycosides and tetracyclines) leading to false evaluations during laboratory screening. ${ }^{26}$ In this regard, the LTM is superior, as antibiotics will be uniformly distributed and for the antibodies with a similar half-life, the MIC will truly reflect their efficacy. Modifications in the dosage for DDM may also be warranted because of the nature of clinical isolates, which, even though are E. coli, may have differently adapted to harsher conditions in the host and environment when compared with the laboratory strains used for determining the dosage on the disc. Our reasoning was substantiated by the fact that, when we doubled the antibiotic dose to counter the above limitations, we found that the results matched well with those obtained using LTM. Therefore, in the absence of an evaluation of the contributions of these interferences and the properties of antibiotics affecting the zone of clearance, instead of complicated optimization for DDM, the simple adaptation of using two discs or doubling the dose on a disc could be the easiest way to compensate for the anomalies.

Efflux assay coupled with LTM is a desirable diagnostic method The adaptation of efflux by bacteria obviates the need to develop unique resistance mechanisms for individual antibiotics and facilitates resistance to group-specific or to a wide spectrum of antibiotics. ${ }^{6,7}$ Therefore, the identification of even 'intermediary' types becomes important in choosing the right antibiotic. Certain subjectivity in the DDM could vitiate this estimate, as can be seen from this study. The capability of quantitative measurement using LTM provides a better handle to identify such 'intermediary' cases with increased confidence. To enhance the scope of such a possibility, we tested the use of a welldocumented marker dye, FDA, to detect efflux phenotype during the determination of antibiogram. ${ }^{21,23}$ Accordingly, the fluorescence resulting from the uptake of non-fluorescent FDA and efflux as fluorescein by viable and growing cells indicates efflux pump-dependent resistance. This observation correlated well with the antibiogram results obtained from the modified DDM and LTM methods.

Potential of LTM as a clinical tool for antibiogram determination In conclusion, LTM, a potential tool for tackling diseases caused by MDR pathogens, can be performed within $7-8 \mathrm{~h}$ as against $2-3$ days for DDM. It is also easily performed in 96-well microtiter plates, routinely used in clinical practice and in MIC determination. ${ }^{19}$ Inclusion of the efflux test by a convenient and established fluorescent method, as used in this study, will not only substantiate the MDR phenotype but will also indicate the mechanism involved. The occurrences of some false positives are possible, if the efflux pumps or efficiency for the dye and the drug are different. The LTM can be easily adopted in clinical laboratories, including peripheral hospitals. It is also economical from the view of labor, use of antibiotics and media, as well as waste generated. Being suitable for high-throughput format, it is highly compatible for instrumentation in surveillance and epidemiology. A significant outcome of this study is the fact that, in accordance with the recent observation and reports of clinicians, E. coli isolates were in fact 'resistant' to multiple antibiotics, especially to third-generation cephalosporins $(\sim 65 \%)$, but antibiotics such as Gentamycin and Amikacin are still effective.

\section{ACKNOWLEDGEMENTS}

We acknowledge Dr Jayanthi, Dr Saradha Suresh, Dr Md Meeran and Dr Umadevi of the Institute of Child Health and Hospital for Children (ICH and $\mathrm{HC}$ ), Chennai, India for their support in sample collection and discussions. We thank Dr Rolf Reissbordt for contributing reference EPEC strains. We gratefully acknowledge the financial support from the Department of Science and Technology, India under their Technology Development Programme, UGC under DRS as well as the Centre with Potential for Excellence Programme and European Union-sponsored Each Child project.

1 McCormick, J. B. Epidemiology of emerging/re-emerging antimicrobial-resistant bacterial pathogens. Curr. Opin. Microb. 1, 125-129 (1998).

2 US-FDA website: http://www.fda.gov/ola/2008/antimicrobials (13 December 2008, date last accessed).

3 Giske, C. G., Dominique, L., Cars, M. O. \& Carmeli, Y. Clinical and economic impact of common multidrug-resistant Gram-negative Bacilli. Antimicrob. Agents Chemother. 52, 813-821 (2008)

4 Bradford, P. A. Extended spectrum $\beta$-Lactamases in 21st century: characterization, epidemiology, and detection of this important resistance thereat. Clin. Microbiol. Rev. 14, 933-951 (2001).

5 Nikaido, H. Multi-drug efflux in bacteria as a basis of resistance. Abstr. Intersci. Conf. Antimicrob. Agents Chemother., Intersci. Conf. Antimicrob. Agents Chemother 39, 26-29 (1999)

6 Pitout, J. D. D., Nordmann, P., Laupland, K. B. \& Poirel, L. Emergence of Enterobacteriaceae producing extended-spectrum $\beta$-lactamases (ESBLs) in the community. J. Antimicrob. Chemother. 56, 52-59 (2005).

7 Livermore, D. M. Bacterial resistance: origins, epidemiology, and impact. Clin. Infect. Dis. 36, S11-S23 (2003).

8 Burrus, V., Pavlovic, G., Decaris, B. \& Guedon, G. Conjugative transposons: the tip of the iceberg. Mol. Microbiol. 46, 601-610 (2002).

9 McDermott, P. F., Walker, R. D. \& White, D. G. Antimicrobials: modes of action and mechanisms of resistance. Int. J. Toxicol. 22, 135-143 (2003).

10 Travers, K. \& Barza, M. Morbidity infections caused by antimicrobial resistant bacteria. Clin. Infect. Dis. 34(Suppl. 3S), 131-134 (2002).

11 Tellefson, L., Fedorka-Cray, P. J. \& Angulo, F. J. Public health aspects of resistance monitoring in the USA. Acta. Vet. Scand. Suppl. 92, 67-75 (1999).

12 Pavankumar, A. R. \& Sankaran, K. The need and new tools for surveillance of Escherichia coli pathogens. Food Technol. Biotechnol. 46, 125-145 (2008).

13 WHO Fact Sheet 2005. http://www.oneworldhealth.org (13 December 2008, date last accessed) 
14 Mayer, R. M. \& Koshi, G. Manual of Diagnostic Procedures in Medical Microbiology and Immunology/Serology, Revised edn 70-75 (Faculty, Christian Medical College and Hospital, Vellore, India, 2001).

15 James, G. C. \& Natalie, S. Microbiology, A Laboratory Manual, 3rd edn (Benjamin/ Cummings Publishing Company, Redwood City, CA, USA, 2005).

16 Kong, R. Y. C., So, C. L., Law, W. F. \& Wu, R. S. S. Asensitive and versatile multiples PCR system for rapid detection of enterotoxigenic, enterohaemorrhagic and enteropathogenic strains of Escherichia coli. Mar. Pollut. Bull. 38, 1207-1215 (1999).

17 Lalitha, M. K. et al. E-test as an alternative to conventional MIC determination for surveillance of drug resistant S. pneumoniae. Indian J. Med. Res. 106, 500-503 (1997).

18 Segal, I. H. Biochemical Calculations, 2nd edn (John Wiley \& Sons Inc., Singapore, 1994).

19 Clinical and Laboratory Standards Institute. Performance Standards for Antimicrobial Susceptibility Testing: Fifteenth Informational Supplement M100-S15 (CLSI, Wayne, PA, 2006).
20 Breeuwer, P., Jean-Louis, D. \& Bunschoten, N. Characterization of uptake and hydrolysis of fluorescein diacetate and carboxyfluorescein diacetate by intracellular esterases in Saccharomyces cerevisiae, which result in accumulation of fluorescent product. App. Environ. Microbiol. 61, 1614-1619 (1995).

21 Lindhagen, E., Nygren, P. \& Larsson, R. The fluorometric microculture cytotoxicity assay. Nat. Protoc. 3, 1364-1369 (2008).

22 Clarke, J. M., Gillings, M. R., Altavilla, N. \& Beattie, A. J. Potential problems with fluorescein diacetate assays of cell viability when testing natural products for antimicrobial activity. J. Microbiol. Meth. 46, 261-267 (2001).

23 Helen, I., Zgurskaya, H. I. \& Nikaido, H. Multi-drug resistance mechanisms: drug efflux across two membranes. Mol. Microb. 37, 219-225 (2000).

24 British Pharmacopoeia TSO associated publications UK (2004).

25 United States Pharmacopoeia. Rockville, MD, USA (2002).

26 National Committee for Clinical Laboratory Standards. Performance Standards for Antimicrobial Testing: Twelfth Informational Supplement (NCCLS, Wayne, PA, USA, 2003). 